\title{
Using higher-order statistics to infer random forces driving a nanosensor
}

\author{
Mohammad Soltani ${ }^{1}$ and Abhyudai Singh ${ }^{1}$
}

\begin{abstract}
Cantilever-based nanosensors are increasingly being used as gas and chemical sensors. We mechanistically model an important source of stochasticity arising in these systems - the random collisions between the sensor and the surrounding molecules. The sensor dynamics is modeled as an underdamped second-order system with molecular collisions occurring at random times, and each strike adding noise to the sensor velocity. The time between two molecular strikes is drawn from an arbitrary distribution. Our analysis of the steady-state variance in the sensor displacement and velocity reveals two insights: firstly, the variance cannot discriminate between frequent low-impact molecular collisions versus rare high-impact collisions. Secondly, the variance only depends on the mean inter-strike time and is completely independent of other properties of the inter-strike time distribution. We next derive higher-order statistical moments (such as kurtosis) of the sensor displacement/velocity and show that these can be harnessed to discriminate between frequency low-impact strikes and rare high-impact strikes. As expected, these moments converge to Gaussian statistics in the limit of frequent molecular strikes. In summary, our work generalizes Brownian noisedriven models and quantifies deviations from Gaussian statistics that arise from mechanistic modeling of physical collisions. Furthermore, higher-order statistics can be exploited to infer random forces impinging on these nanosystems that ultimately determine the sensor signal-to-noise ratio.
\end{abstract}

\section{INTRODUCTION}

Stochastic Hybrid Systems (SHS) constitute an important class of mathematical models that integrate discrete stochastic events with continuous dynamics. Given their generality and scope, SHSs have been successfully used for modeling stochastic phenomena in a variety of systems [1]-[4]. Here we use this versatile framework to explore the stochastic dynamics of a nanosensor with random collisions from surrounding gas molecules.

Nanomechanical resonators are increasingly being used for diverse applications such as atomic force microscope tips, and as force, position, mass, and chemical sensors [5]-[18]. A major source of noise in these systems is the random collisions between surrounding gas molecules and the sensor (Fig. 1). These sensors are often kept in rarefied atmospheres, where the time interval between collisions can be long enough for model approximations based on Brownian noise to fail [19].

Here we recast this problem as an SHS where sensor dynamics follows a linear dynamical system based on a mass-

\footnotetext{
${ }^{1}$ Mohammad Soltani and Abhyudai Singh are with the Departments of Electrical and Computer Engineering, Biomedical Engineering at the University of Delaware, Newark, DE 19716, USA. msoltani@udel.edu, absingh@udel.edu. This work was funded by grants from the Army Research Office (W911NF1910243) and the National Science Foundation (ECCS-1711548) to AS.
}

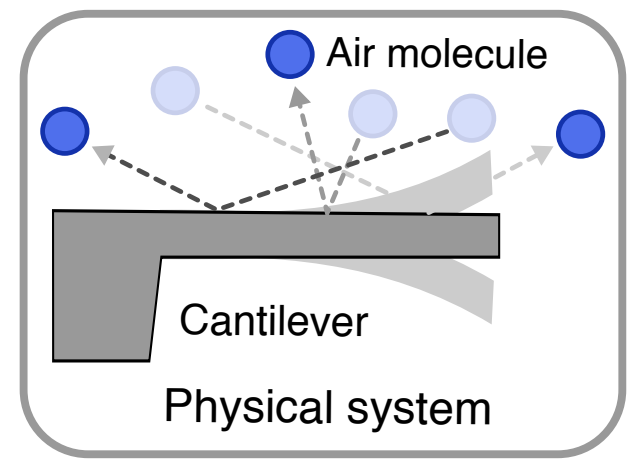

Fig. 1. Schematic of a nanosensor shown as a cantilever beam. Collisions with surrounding gas particles create noisy perturbations in the sensor velocity. Our goal is to investigate how the steady-state distribution of the sensor displacement/velocity are impacted by the physical properties of the collision, such as the frequency of strikes and the impact of each strike.

spring formulation. Molecular collisions occur at discrete random times creating stochastic perturbation in the sensor velocity. Our analysis predicts the extent of fluctuations in the sensor displacement/velocity as a result of these collisions. We further extend these results to look at skewness and kurtosis of the sensor displacement and find that such higher-order statistical measures contain useful information to characterize the system. More specifically, while both frequent lowimpact molecular collisions and rare high-impact collisions result in the same variance in the sensor displacement, both scenarios yield distinct displacement distributions that are reflected in different higher-order moments. Thus, measurement of kurtosis when combined with variance effectively estimates both the frequency of strikes and the impact of each strike. We systematically explore parameter regimes where state variables follow Gaussian statistics. Our analysis identifies regimes where deviations from Gaussian statistics become prominent, and such signatures can be used to characterize the physical properties of molecular collisions.

We start by providing a mathematical formulation of the problem in Section II and develop analytical expressions for lower-order and higher-order moments in Section III and IV, respectively.

\section{MOdeling NANOSENSOR DYNAMICS AS A STOCHASTIC HYBRID SYSTEM}

Given the cantilever-shaped design of several nanosensors [20]-[23], we consider a simple mass-spring formulation where the dynamics are captured by an underdamped secondorder system. The sensor displacement $(x)$ and velocity $(v)$ 
evolve according to the linear dynamical system

$$
\begin{aligned}
& \frac{d x}{d t}=v(t), \\
& \frac{d v}{d t}=-\omega_{n}^{2} x(t)-2 \zeta \omega_{n} v(t),
\end{aligned}
$$

with natural frequency $\omega_{n}$ and damping ratio $\zeta<1$. This continuous-time evolution is interspersed by molecular collisions that create random perturbations in the sensor velocity. Let collisions occus at times $t_{s}, s \in\{1,2, \ldots\}$ and the time interval between collisions (i.e., the inter-strike times)

$$
T \equiv t_{s}-t_{s-1},
$$

are assumed to be independent and identically distributed random variables that follow a continuous positively-valued probability density function $p$. While in the simplest scenario the inter-strike times follow a memoryless exponential distribution, here we decided to keep things general by considering an arbitrary distribution. Whenever collisions occur, the velocity resets as

$$
v \mapsto v+\eta,
$$

where $\eta$ is drawn from an arbitrary distribution with zero mean and variance $\sigma^{2}$. Intuitively, the concentration of molecules determines the frequency of strikes, and $\sigma^{2}$ depends on the mass and velocity of the impinging gas molecules (which in turn is determined by the temperature). The reset (3) can also be written an

$$
v\left(t_{s}^{+}\right)=v\left(t_{s}^{-}\right)+\eta,
$$

where $t_{s}^{-}$and $t_{s}^{+}$denote the time just before and after a collision, respectively. Using (4), one can relate the mean and varince of $v\left(t_{s}^{+}\right)$to the velocity $v\left(t_{s}^{-}\right)$just before the collision

$$
\left\langle v\left(t_{s}^{+}\right)\right\rangle=v\left(t_{s}^{-}\right), \quad\left\langle v^{2}\left(t_{s}^{+}\right)\right\rangle-\left\langle v\left(t_{s}^{+}\right)\right\rangle^{2}=\sigma^{2} .
$$

Here and throughout the paper we use the symbol \langle\rangle to denote the expected value operation.

A convenient approach to model the timing of events is via a timer $\tau$ that measures the time elapsed since the last collision. The timer increases with time

$$
\frac{d \tau}{d t}=1
$$

in between events and resets to zero whenever the events occur. Let the probability that a molecular collision event occurs in an infinitesimal time $(t, t+d t]$ be given by $h(\tau) d t$, where

$$
h(\tau) \equiv \frac{p(\tau)}{1-\int_{y=0}^{\tau} p(y) d y} .
$$

Then, the duration between events $T$ follows a probability density function $p$ given by

$$
T \sim p(\tau)=h(\tau) \mathrm{e}^{-\int_{0}^{\tau} h(y) d y}
$$

[24], [25]. Note that a constant rate $h=1 /\langle T\rangle$ would correspond to a Poisson arrival process where inter-strike times are exponentially distributed with mean $\langle T\rangle$. Similarly,

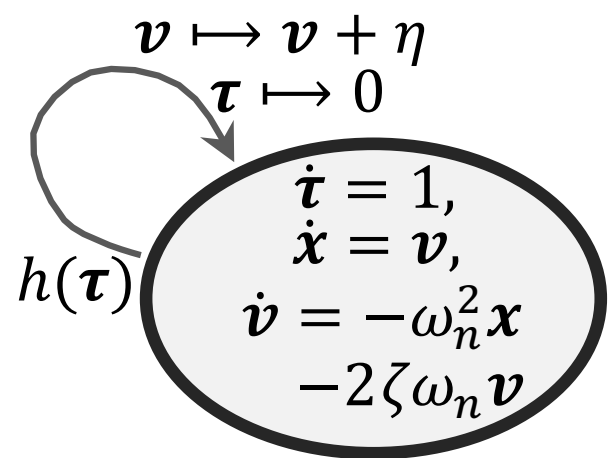

Fig. 2. SHS framework to capture the stochastic dynamics of the nanosensor with impinging gas molecules. The sensor displacement $(x)$ and velocity $(v)$ evolve according to a second-order linear dynamical system with natural frequency $\omega_{n}$ and damping ratio $\zeta$. Timing of molecular strikes is modeled via a timer $\tau$, and whenever the strike occurs the sensor velocity changes as per the reset shown at the end of the arrow. The strikes occur as per an inhomogeneous Poisson process with a rate given by (7), which ensures that the inter-strike times are independent and identically distributed random variables that follow an arbitrary probability distribution $p$.

$h(\tau) \propto \tau^{\alpha}$ leads to a Weibull distributed inter-strike times, with $\alpha>0(\alpha<0)$ corresponding to scenarios where the probality of occurence of the next event increases (decreases) as more time elaspes since the previous event [26].

The overall system as given by equations (1)-(8) is illustrated in Fig. 2 with deterministic dynamics shown within the circle, along with molecular strikes that occur randomly with rate $h(\tau)$ resetting the timer and the sensor velocity. It is important to point out that the model in Fig. 2 falls into the class of piecewise-deterministic Markov processes [27] that has been referred to in the literature as timetriggered Stochastic Hybrid Systems (SHS) [28]-[30]. Such time-triggered SHS have found tremendous applications in modeling stochastic dynamics of engineering and biological systems [31]-[41].

\section{STEADY-STATE VARIANCE OF SENSOR DISPLACEMENT AND VELOCITY}

Having defined the stochastic dynamical system in Fig. 2, we first focus our attention on the first- and second-order statistical moments of the random processes $x(t)$ and $v(t)$. Using well-known tools for deriving moment dynamics [42][44], we obtain the following result: for any continuously differentiable function $\varphi(x, v)$, the time evolution of its expected value is given by

$$
\begin{aligned}
\frac{d\langle\varphi(x, v)\rangle}{d t}= & \left\langle\Delta \varphi(x, v) \times h(\tau)+\frac{\partial \varphi(x, v)}{\partial x} v(t)\right\rangle \\
& +\left\langle\frac{\partial \varphi(x, v)}{\partial v}\left(-\omega_{n}^{2} x(t)-2 \zeta \omega_{n} v(t)\right)\right\rangle,
\end{aligned}
$$

where

$$
\Delta \varphi(x, v)=\varphi(x, v+\eta)-\varphi(x, v)
$$

is the change in $\varphi$ when a collision occurs. Time evolution of all moments up to order 2 can be obtained by simply 
choosing $\varphi(x, v)$ to be $x, v, x^{2}, x v, v^{2}$ in (9) that yields the following linear system of differential equations

$$
\begin{aligned}
& \frac{d\langle x\rangle}{d t}=\langle v\rangle, \\
& \frac{d\langle v\rangle}{d t}=-\omega_{n}^{2}\langle x\rangle-2 \zeta \omega_{n}\langle v\rangle, \\
& \frac{d\left\langle x^{2}\right\rangle}{d t}=2\langle x v\rangle, \\
& \frac{d\langle x v\rangle}{d t}=\left\langle v^{2}\right\rangle-\omega_{n}^{2}\left\langle x^{2}\right\rangle-2 \zeta \omega_{n}\langle x v\rangle, \\
& \frac{d\left\langle v^{2}\right\rangle}{d t}=-2 \omega_{n}^{2}\langle x v\rangle-4 \zeta \omega_{n}\left\langle v^{2}\right\rangle+\langle h(\tau)\rangle \sigma^{2} .
\end{aligned}
$$

Recall that \langle\rangle denotes the expected value and we now introduce another notation $\overline{\langle\rangle}$ to represents the steady-state moments, i.e., $\overline{\langle x\rangle} \equiv \lim _{t \rightarrow \infty}\langle x(t)\rangle$. Solving the linear system (11) at steady-state and using the fact that

$$
\langle T\rangle=\frac{1}{\langle h(\tau)\rangle}
$$

[31], [33], [45], results in the trivial result of zero average sensor displacement and velocity $(\overline{\langle x\rangle}=\overline{\langle v\rangle}=0)$ together with the variances

$$
\begin{aligned}
& \operatorname{var}(x) \equiv \overline{\left\langle x^{2}\right\rangle}-\overline{\langle x\rangle^{2}}=\frac{\sigma^{2}}{4\langle T\rangle \zeta \omega_{n}^{3}} \\
& \operatorname{var}(v) \equiv \overline{\left\langle v^{2}\right\rangle}-\overline{\langle v\rangle^{2}}=\frac{\sigma^{2}}{4\langle T\rangle \zeta \omega_{n}} .
\end{aligned}
$$

Intriguingly, (13) shows that the noise is inversely dependent on $\langle T\rangle$ and invariant of higher-order moments of $T$. Thus, counterintuitively, making timing of collisions more random (for a fixed $\langle T\rangle$ ) will have no impact on $\left\langle x^{2}\right\rangle$ and $\left\langle v^{2}\right\rangle$. Furthermore, measurements of $\left\langle x^{2}\right\rangle$ and $\left\langle v^{2}\right\rangle$ are proportional to the ratio $\sigma^{2} /\langle T\rangle$, and hence cannot discriminate between infrequent high-impact strikes (large $\sigma^{2}$ and $\langle T\rangle$ ), and frequent low-impact strikes (small $\sigma^{2}$ and $\langle T\rangle$ ). Finally, note from (11c) that $\overline{\langle x v\rangle}=0$ resulting in uncorrelated sensor displacement and velocity.

We next present an analytical study of the autocorrelation function (normalized by the steady-state variance) that is defined as

$$
R_{x}(t) \equiv \lim _{s \rightarrow \infty} \frac{\overline{\langle x(t+s) x(s)\rangle}-\overline{\langle x\rangle}^{2}}{\overline{\left\langle x^{2}\right\rangle}-\overline{\langle x\rangle}^{2}} .
$$

To progress further we use the relation

$$
\langle x(t+s) x(s)\rangle=\langle x(s)\langle x(t+s) \mid x(s)\rangle\rangle,
$$

where $\langle x(t+s) \mid x(s)\rangle$ is the expected value of $x$ at time $t+s$ given $x(s)$. The approach of determining $R_{x}(t)$ is outlined in [46], [47] and relies on solving the moment dynamic equations (11) assuming deterministic initial conditions of $x(s)$ and $v(s)$. Solving the moment dynamics yields the conditional expectation $\langle x(t+s) \mid x(s)\rangle$ that is unconditioned as per (15) to obtain $\langle x(t+s) x(s)\rangle$. Using this approach we

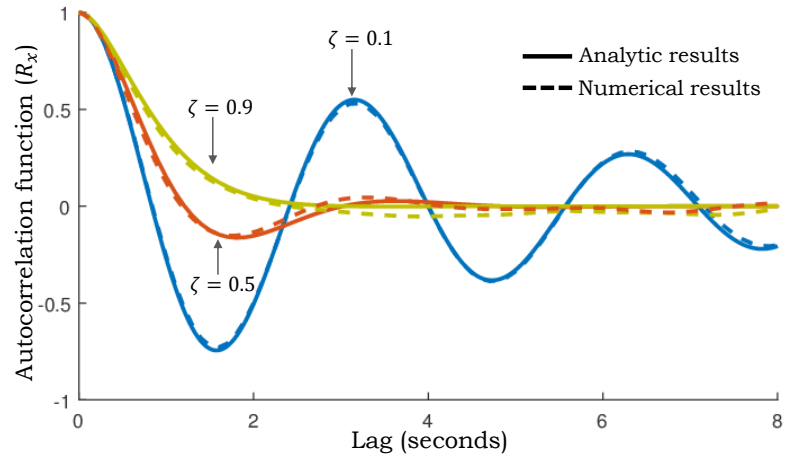

Fig. 3. The autocorrelation function of sensor displacement changes qualitatively with the damping ratio $\zeta$. The autocorrelation function (16) is plotted for different values of $\zeta$ and becomes more oscillatory with decreasing damping. While the autocorrelation function does not provide any information for inferring collision-time statistics it can be used to infer $\zeta$ and $\omega_{n}$. The analytically predicted autocorrelation function matched well with that obtained numerically from Monte Carlo simulation trajectories of the SHS. For this plot natural frequency is selected to be $\omega_{n}=2 \mathrm{rad} / \mathrm{sec}$ and the numerical results are tested over different $\sigma$ considering exponentially distributed collision times.

obtain the following normalized autocorrelation function of sensor displacement

$$
\begin{aligned}
& R_{x}(t) \\
& =e^{-\omega_{n} \zeta t}\left(\frac{\zeta^{2} \sinh \left(\omega_{n} \sqrt{\zeta^{2}-1} t\right)}{\sqrt{\zeta^{2}-1}}+\cosh \left(\omega_{n} \sqrt{\zeta^{2}-1} t\right)\right),
\end{aligned}
$$

and an exactly similar process can be used to obtain the autocorrelation function of velocity. Note that these normalized autocorrelation functions are completely independent of the timing and strength of the strikes, and only depend on the natural frequency $\omega_{n}$ and damping ratio $\zeta$. Plots of $R_{x}(t)$ are illustrated in Fig. 3 for varying $\zeta$ that match with numerically computed autocorrelation functions.

So far we have observed that for a given mean collision time $\langle T\rangle$, the specific distribution of collision times does not impact the mean, variance, autocorrelation function of sensor displacement and velocity. However, performing Monte Carlo simulations of the time-triggered SHS shows that the shape of the displacement's distribution is indeed impacted by the distribution of $T$ (Fig. 4). This motivates the computations of higher-order moments that may contain additional information to infer model parameters.

\section{QUANTIFYING HIGHER-ORDER STATISTICAL MOMENTS}

Since collisions on the sensor are assumed to occur in both directions, the resulting zero-mean steady-state distributions of the sensor displacement and velocity are symmetric (Fig. 4) with zero skewness

$$
\overline{\left\langle x^{3}\right\rangle}=0, \quad \overline{\left\langle v^{3}\right\rangle}=0 .
$$




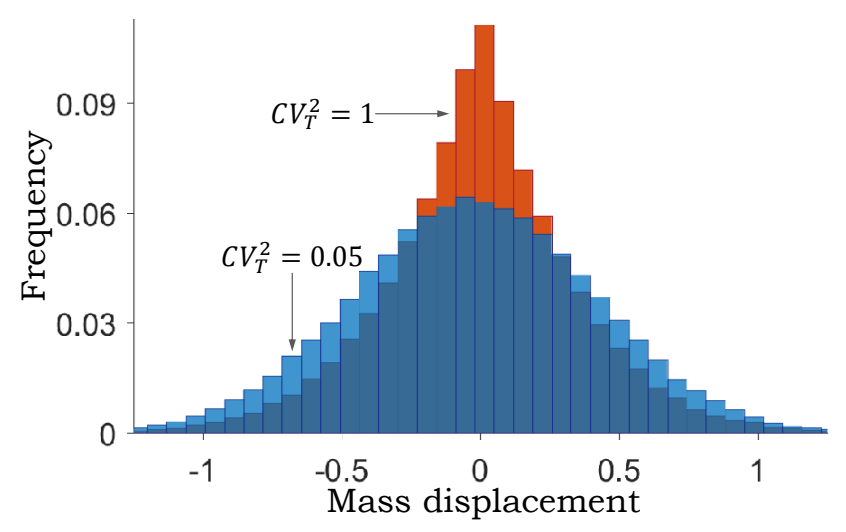

Fig. 4. Noise in collision arrival times changes the shape of the displacement distribution. Distribution of the sensor displacement $x(t)$ as obtained by performing 10,000 Monte Carlo simulations of the timetriggered SHS in Fig. 2 for $\omega_{n}=2 \mathrm{rad} / \mathrm{sec}, \zeta=0.1,\langle T\rangle=1 \mathrm{sec}$, and $\sigma=0.5$. Here $C V_{T}$ represents the noise in the inter-strike times $T$ as quantified by its coefficient of variation (standard deviation divided by the mean) and the distribution is plotted for two cases: exponentially distributed $\left(C V_{T}^{2}=1\right)$ and gamma distributed $T\left(C V_{T}^{2}=0.05\right)$. While both scenarios result in the same steady-state mean and variance in the sensor displacement, increasing $C V_{T}$ makes the distribution more "peaked" which can be quantified by higher-order statistical measures, such as kurtosis.

We next focus our attention on the fourth-order moment commonly known as kurtosis

$$
\text { Kurtosis of } x=\frac{\overline{\left\langle x^{4}\right\rangle}}{{\overline{\left\langle x^{2}\right\rangle}}^{2}}, \quad \text { Kurtosis of } v=\frac{\overline{\left\langle v^{4}\right\rangle}}{\overline{\left\langle v^{2}\right\rangle}}
$$

that is often used to quantify the "peakedness" of a probability distribution. The kurtosis of a Gaussian distribution is 3 , and excess kurtosis is defined as kurtosis minus 3 .

Recall that the random variable $\eta$ determines the perturbation in sensor velocity upon collision (Fig. 2). Let

$$
\beta=\frac{\overline{\left\langle\eta^{4}\right\rangle}}{\overline{\left\langle\eta^{2}\right\rangle}}
$$

be the kurtosis of $\eta$, which implies that

$$
\begin{aligned}
& \beta=\frac{\left\langle\left(v\left(t_{s}^{+}\right)-\left\langle v\left(t_{s}^{+}\right)\right\rangle\right)^{4}\right\rangle}{\left\langle v^{2}\left(t_{s}^{+}\right)-\left\langle v\left(t_{s}^{+}\right)\right\rangle^{2}\right\rangle^{2}} \\
& \Rightarrow\left\langle v^{4}\left(t_{s}^{+}\right)\right\rangle=\beta \sigma^{4}+6 \sigma^{2} v\left(t_{s}^{-}\right)^{2}+v^{4}\left(t_{s}^{-}\right) .
\end{aligned}
$$

Typically, $\beta=3$ assuming that the velocity of impinging molecules is normally distributed. It is important to point out here that detailed physics-based calculations can be done to relate both $\sigma^{2}$ and $\eta$ to the properties of the colliding particles. This can be done by considering that their velocity follows a Maxwell-Boltzman distribution with an average speed that is proportional to the square root of the ratio of temperature and particle mass [48], [49].

While the method of moment dynamics as illustrated in equations (9)-(11) works well for quantifying lower-order moments, it fails when analyzing fourth-order moments given the nonlinear rate $h(\tau)$. Except for the case of exponentially distributed stikes for which $h=1 /\langle T\rangle$ is a constant, nonlinearities in reset rates lead to an unclosed system of moment equations that are often solved by employing approximate closure schemes [50]-[58].

We have recently introduced an alternative analytical framework for exact quantification of statistical moments for time-triggered SHS with linear continuous dynamics [33]. Here, the state-space is augmented by defining a new vector that has all monomials of $x$ and $v$ of second, third and fourth order

$$
\mu=\left[\begin{array}{llllllll}
x^{2} & v^{2} & x v & x^{4} & v^{4} & x^{3} v & x^{2} v^{2} & x v^{3}
\end{array}\right]^{\top} .
$$

Dynamics of $\mu$ in between successive collision events is given by the linear system

$$
\dot{\mu}=A_{\mu} \mu, A_{\mu} \equiv\left[\begin{array}{cc}
A_{1} & 0_{3 \times 3} \\
0_{5 \times 3} & A_{2}
\end{array}\right],
$$

where $0_{3 \times 3}$ and $0_{5 \times 3}$ denote zero matrices of appropriate dimensions, and

$$
\begin{aligned}
A_{1} & =\left[\begin{array}{ccc}
0 & 0 & 2 \\
0 & -4 \zeta \omega_{n} & -2 \omega_{n} \\
-\omega_{n}^{2} & 1 & -2 \zeta \omega_{n}
\end{array}\right] \\
A_{2} & =\left[\begin{array}{ccccc}
0 & 0 & 4 & 0 & 0 \\
0 & -8 \zeta \omega_{n} & 0 & 0 & -4 \omega_{n}^{2} \\
-\omega_{n}^{2} & 0 & -2 \zeta \omega_{n} & 3 & 0 \\
0 & 0 & -2 \omega_{n}^{2} & -4 \zeta \omega_{n} & 2 \\
0 & 1 & 0 & -3 \omega_{n}^{2} & -6 \zeta \omega_{n}
\end{array}\right] .
\end{aligned}
$$

At the time of collision, the reset in velocity $v \mapsto v+\eta$ can be used to determine the corresponding reset in $\mu$. Using (5) and (20), the expected value of $\mu$ just after collision can be related to its value just before collision as

$$
\left\langle\mu\left(t_{s}^{+}\right)\right\rangle=J_{\mu} \mu\left(t_{s}^{-}\right)+R_{\mu},
$$

where elements of $J_{\mu} \in \mathbb{R}^{8 \times 8}$ and $R_{\mu} \in \mathbb{R}^{8 \times 1}$ are

$\left(J_{\mu}\right)_{i j} \equiv\left\{\begin{array}{cl}1 & i=j, \\ 6 \sigma^{2} & i=5, j=2, \\ \sigma^{2} & i=7, j=1, \\ 0 & \text { otherwise }\end{array} \quad,\left(R_{\mu}\right)_{i} \equiv\left\{\begin{array}{cl}\sigma^{2} & i=2, \\ \beta \sigma^{4} & i=5, \\ 0 & \text { otherwise }\end{array}\right.\right.$

In essence, by augmenting the state-space we have defined a new time-triggered SHS whose state-space $\mu$ follows the continuous dynamics (22) with reset (24) that occurs at collision times. Applying Theorem 3.1 in [33] provides explicit conditions for the existence of $\overline{\langle\mu\rangle}$ along with an analytical expression for it. We use this result to derive all steady-state moments of $x$ and $v$ up to order 4 that are then used to obtain the kurtosis as defined in (18).

Performing the analysis as outlined above yields the following steady-state kurtosis for sensor displacement

$$
\frac{\overline{\left\langle x^{4}\right\rangle}}{{\overline{\left\langle x^{2}\right\rangle}}^{2}}=\frac{3 \beta\langle T\rangle \omega_{n} \zeta}{6 \zeta^{2}+2}+\frac{6 \omega_{n} \zeta\langle T\rangle\left\langle e^{-2 \omega_{n} \zeta T}\right\rangle}{1-\left\langle e^{-2 \omega_{n} \zeta T}\right\rangle} .
$$

Studying this result sheds interesting insights:

- The kurtosis is independent of $\sigma^{2}$ and can be decomposed into two parts.

- The first-term on the right-hand-side of (26) representing the contribution from $\beta$. The second term is 

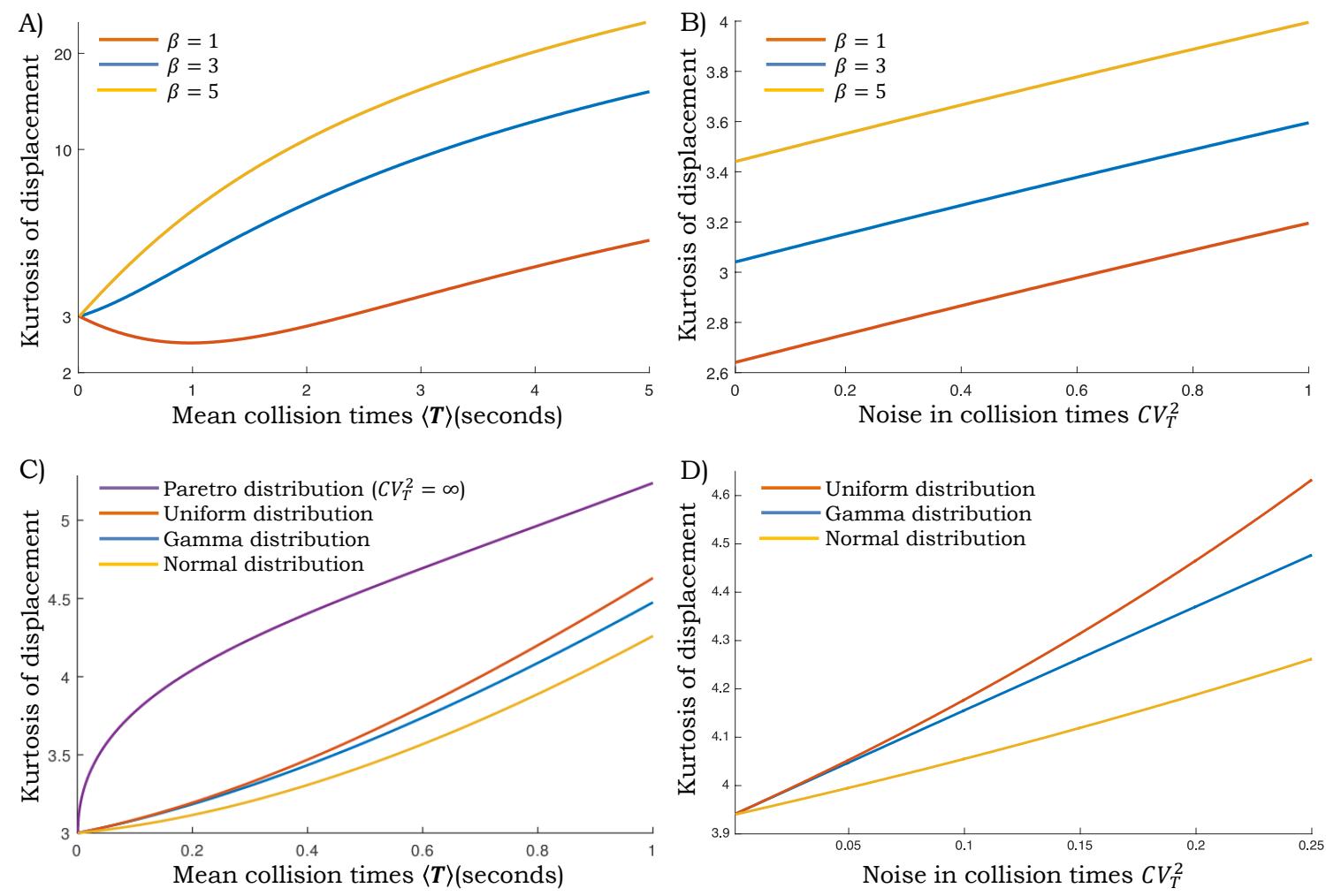

Fig. 5. The steady-state kurtosis of sensor displacement depends on the statistical characteristics of collision times. A) In the limit of $\langle T\rangle \approx 0$, kurtosis is 3 for any values of $\beta$. Kurtosis can be less than 3 for $\beta<3$ and intermediate collision times. Kurtosis grows unboundedly as $\langle T\rangle \rightarrow \infty$. B) Kurtosis monotonically increases with increasing $C V_{T}^{2}$, where $C V_{T}$ is the coefficient of variation of $T$. However, kurtosis saturates to a finite value as $C V_{T}^{2} \rightarrow \infty$. For both A and B, time intervals are gamma-distributed with $C V_{T}^{2}=0.25$ in A and $\langle T\rangle=1$ sec in B. C \& D) Different collision-time distributions results in the similar qualitative behavior in kurtosis. Note that we selected a Pareto distribution with $C V_{T}^{2}=\infty$, hence for any mean collision time the value of this plot shows the upper limit of kurtosis as $C V_{T}^{2} \rightarrow \infty$. For both $\mathrm{C}$ and $\mathrm{D}, \beta$ is 3 . For all the plots the rest of the parameters are selected as $\omega_{n}=2 \mathrm{rad} / \mathrm{sec}$ and $\zeta=0.5$.

the contribution from random timing of strikes that is related to the moment generating function of $T$.

- In the limit of frequency strikes $(T \rightarrow 0)$, the kurtosis reduces to 3 consistent with Gaussian statistics. This limit can be intuitively thought of as averaging over a large number of noisy molecular hits resulting in a Gaussian distribution from the the central limit theorem.

- For exponentially distributed collision times

$$
\left\langle e^{-2 \omega_{n} \zeta T}\right\rangle=\frac{1}{1+2 \omega_{n} \zeta\langle T\rangle}
$$

and (26) reduces to

$$
\frac{\overline{\left\langle x^{4}\right\rangle}}{{\overline{\left\langle x^{2}\right\rangle}}^{2}}=3+\frac{3 \beta\langle T\rangle \omega_{n} \zeta}{6 \zeta^{2}+2}
$$

with $\frac{3 \beta\langle T\rangle \omega_{n} \zeta}{6 \zeta^{2}+2}$ representing the excess kurtosis. A similar formula is obtained for kurtosis of velocity $v$

$$
\frac{\overline{\left\langle v^{4}\right\rangle}}{\overline{\left\langle v^{2}\right\rangle}}=3+\frac{3 \beta\langle T\rangle \omega_{n} \zeta\left(1+4 \zeta^{2}\right)}{6 \zeta^{2}+2}
$$

which is predicted to be larger than the kurtosis of displacement.

In Fig. 5 we illustrate the kurtosis as a function of $\beta$, mean strike-time $\langle T\rangle$, noise in collision times $C V_{T}$ (as quantified by the coefficient of variation of $T$ ), and for a variety of different distributions of $T$. The plot shows an increasing trend of kurtosis with increasing $\langle T\rangle, C V_{T}$, and $\beta$. This point is also exemplified in Fig. 6, where for $\langle T\rangle \rightarrow 0$ kurtosis is always 3 irrespective of $C V_{T}$, but increases monotonically with respect to both $\langle T\rangle$ and $C V_{T}$. Since kurtosis is a measure of peakedness, a higher kurtosis means that the random variable is more centered around the steady-state - this can be seen in Fig. 4 where the case of $C V_{T}^{2}=1$ has a higher kurtosis compared to $C V_{T}^{2}=0.05$ with both distributions having the same variance. The analysis in Fig. 5 shows that across parameter regimes the excess kurtosis is mostly positive. Interestingly, a negative excess kurtosis is possible in the case of $\beta<3$ and $C V_{T}^{2}<1$, where the kurtosis dips below 3 with increasing $\langle T\rangle$ and then increases with increasing $\langle T\rangle$ (Fig. 5A). Moreover, changing the distribution of collision times does not qualitatively change the behavior of kurtosis as shown in Fig. 5C and Fig. 5D.

\section{Discussion AND Future Work}

In this contribution, we have used an SHS-formalism to mechanistically incorporate the physical collisions of molecules on a cantilever-based nanosensor (Figs. 1 and 2). Analysis of the resulting stochastic dynamical system yielded exact analytical expressions for the state-space vari- 


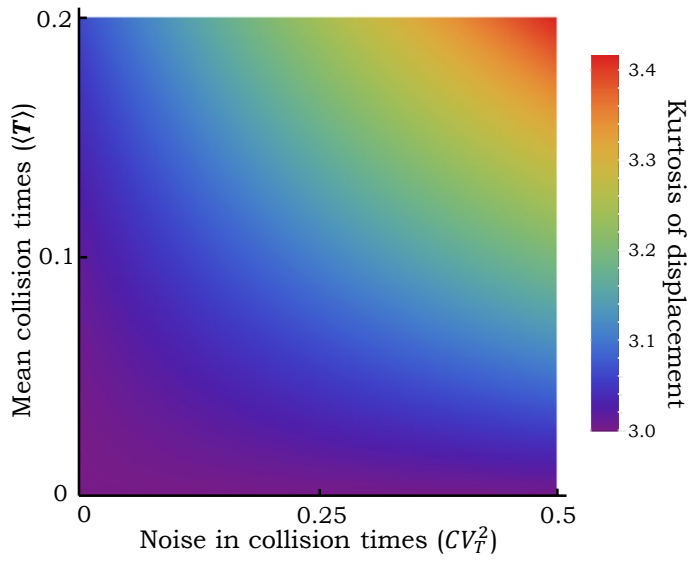

Fig. 6. The steady-state kurtosis of sensor displacement increases monotonically with the mean and noise in collision times. Assuming a gamma distributed inter-strike times $T$, kurtosis as predicted by (26) is plotted as a function of $\langle T\rangle$ and $C V_{T}^{2}$ assuming $\beta=3, \omega_{n}=2 \mathrm{rad} / \mathrm{sec}$ and $\zeta=0.5$. In the limit, $\langle T\rangle \rightarrow 0$ where strikes occur frequently kurtosis converges to 3 irrespective of $C V_{T}$ consistent with that of a Gaussian distribution. As collisions become infreqeunct with increasing $\langle T\rangle$, the variance in the sensor displacement decreases as per (13), but kurtosis increases beyond 3 creating deviations from Gaussian statistics.

ance in the sensor displacement/velocity, along with the autocorrelation function of these random processes. These formulas were in good agreement with numerically obtained counterparts from Monte Carlo simulation runs of the SHS (Fig. 3).

While second-order statistical moments quantify the extent of noise that is induced by collisions, it is proportional to $\sigma^{2} /\langle T\rangle$, and hence cannot discriminate between frequency low-impact strikes versus rare high-impact strikes. We extended the result to derive the fourth-order moments of displacement and velocity by quantifying their steadystate kurtosis. Interestingly, the kurtosis was found to be invariant of $\sigma^{2}$ but depended on $T$ through its moment generating function. Thus, having an a-prior knowledge about system parameters $\left(\omega_{n}, \zeta, \beta\right)$, and assuming exponentially distributed collision times, $\langle T\rangle$ can be inferred from the kurtosis. Our results show convergence to Gaussian statistics in the limit of frequent strikes $(\langle T\rangle \rightarrow 0)$ where the SHS model reduces to the classical model of Brownian noise driving a linear dynamical system. Furthermore, the kurtosis provided rich insights into deviations from Gaussian statistics for different inter-strike time distributions as a function of $\beta$ and $\langle T\rangle$ (Figs. 4-6). While much work on stochastic systems focuses on a few lower-order moments, computation of higher-order moments can inform on system properties, and in this case, disentangle the impacts of $\sigma^{2}$ and $\langle T\rangle$. In the context of a chemical nanosensor, it can be particularly useful to infer $\langle T\rangle$ separately, which in turn would be inversely proportional to the concentration of molecules.

In future work, we will further develop these results and also look at the collision of molecules in two or three dimensions. It will be fascinating to connect these results back to the fundamental work of Robert Brown in 1827, where he observed the irregular zigzag movements of a Pollen grain in water due to molecular collisions [59], [60]. Another interesting avenue is to investigate feedback strategies, such as applying an external force dependent on displacement and/or velocity for mitigating noise in nanosensors. We have recently extended the theory of time-triggered SHS to nonlinear dynamics systems [61], which can be used to explore the effects of nonlinearities in beam deformation and physical collisions on state fluctuations.

\section{REFERENCES}

[1] S. Bohacek, J. P. Hespanha, J. Lee, and K. Obraczka, "A hybrid systems modeling framework for fast and accurate simulation of data communication networks," in Proc. of the ACM Int. Conf. on Measurements and Modeling of Computer Systems (SIGMETRICS), vol. 31, 2003, pp. 58-69.

[2] J. Hu, "Application of stochastic hybrid systems in power management of streaming data," in Proc. of the 2006 Amer. Control Conference, Minneapolis, MN, 2006, pp. 4754-4759.

[3] W. Liu and I. Hwang, "Stochastic hybrid system model with applications to aircraft trajectory prediction and conflict detection," AIAA Journal on Guidance, Dynamics and Control, vol. 34, 2011.

[4] M. Strelec, K. Macek, and A. Abate, "Modeling and simulation of a microgrid as a stochastic hybrid system," in 3rd IEEE PES International Conference and Exhibition on Innovative Smart Grid Technologies (ISGT Europe), 2012, pp. 1-9.

[5] M. Di Ventra, S. Evoy, J. R. Heflin, and D. J. Lockwood, Eds., Introduction to Nanoscale Science and Technology, ser. Nanostructure Science and Technology. Springer US, 2004.

[6] K. M. Goeders, J. S. Colton, and L. A. Bottomley, "Microcantilevers: sensing chemical interactions via mechanical motion," Chemical Reviews, vol. 108, pp. 522-542, 2008.

[7] J. Arlett, E. Myers, and M. Roukes, "Comparative advantages of mechanical biosensors," Nature Nanotechnology, vol. 6, pp. 203-215, 2011

[8] P. S. Waggoner and H. G. Craighead, "Micro- and nanomechanical sensors for environmental, chemical, and biological detection," Lab Chip, vol. 7, pp. 1238-1255, 2007.

[9] A. N. Cleland and M. L. Roukes, "Fabrication of high frequency nanometer scale mechanical resonators from bulk si crystals," Applied Physics Letters, vol. 69, no. 18, pp. 2653-2655, 1996.

[10] H. G. Craighead, "Nanoelectromechanical systems," Science, vol. 290, no. 5496, pp. 1532-1535, 2000.

[11] V. Sazonova, Y. Yaish, H. Üstünel, D. Roundy, T. A. Arias, and P. L. McEuen, "A tunable carbon nanotube electromechanical oscillator," Nature, vol. 431, no. 7006, pp. 284-287, 2004.

[12] K. Ekinci and M. Roukes, "Nanoelectromechanical systems," Review of scientific instruments, vol. 76, no. 6, p. 061101, 2005.

[13] Y.-T. Yang, C. Callegari, X. Feng, K. L. Ekinci, and M. L. Roukes, "Zeptogram-scale nanomechanical mass sensing," Nano letters, vol. 6 , no. 4, pp. 583-586, 2006.

[14] H. Peng, C. Chang, S. Aloni, T. Yuzvinsky, and A. Zettl, "Ultrahigh frequency nanotube resonators," Physical review letters, vol. 97, no. 8, p. 087203, 2006.

[15] R. Raiteri, M. Grattarola, H.-J. Butt, and P. Skládal, "Micromechanical cantilever-based biosensors," Sensors and Actuators B: Chemical, vol. 79, no. 2-3, pp. 115-126, 2001.

[16] E. Buks and B. Yurke, "Mass detection with a nonlinear nanomechanical resonator," Physical Review E, vol. 74, no. 4, p. 046619, 2006.

[17] K. Jensen, K. Kim, and A. Zettl, "An atomic-resolution nanomechanical mass sensor," Nature nanotechnology, vol. 3, no. 9, pp. 533-537, 2008

[18] A. Singh, R. Mukherjee, K. Turner, and S. Shaw, "Mems implementation of axial and follower end forces," Journal of sound and vibration, vol. 286, no. 3, pp. 637-644, 2005.

[19] K. L. Ekinci, Y. T. Yang, and M. L. Roukes, "Ultimate limits to inertial mass sensing based upon nanoelectromechanical systems," Journal of Applied Physics, vol. 95, pp. 2682-2689, 2004.

[20] A. Boisen and T. Thundat, "Design \& fabrication of cantilever array biosensors," Materials Today, vol. 12, pp. 32-38, 2009.

[21] R. Datar, S. Kim, S. Jeon, P. Hesketh, S. Manalis, A. Boisen, and T. Thundat, "Cantilever sensors: Nanomechanical tools for diagnostics," MRS Bulletin, vol. 34, pp. 449-454, 2009. 
[22] A. Boisen, S. Dohn, S. S. Keller, S. Schmid, and M. Tenje, "Cantileverlike micromechanical sensors," Reports on Progress in Physics, vol. 74, p. 036101, 2011.

[23] J. Fritz, "Cantilever biosensors," Analyst, vol. 133, pp. 855-863, 2008.

[24] S. M. Ross, "Reliability theory," in Introduction to Probability Models, 10th ed. Academic Press, 2010, pp. $579-629$.

[25] M. Evans, N. Hastings, and B. Peacock, Statistical Distributions, 3rd ed. Wiley, 2000.

[26] R. Jiang and D. Murthy, "A study of weibull shape parameter: Properties and significance," Reliability Engineering \& System Safety, vol. 96, no. 12, pp. 1619-1626, 2011.

[27] M. H. A. Davis, "Piecewise-deterministic markov processes: A general class of non-diffusion stochastic models," Journal of the Royal Statistical Society. Series B (Methodological), vol. 46, pp. 353-388, 1984.

[28] A. Singh and J. P. Hespanha, "Stochastic hybrid systems for studying biochemical processes," Philosophical Transactions of the Royal Society A, vol. 368, pp. 4995-5011, 2010.

[29] J. Hespanha, "Modelling and analysis of stochastic hybrid systems," IEE Proceedings Control Theory and Applications, vol. 153, pp. 520535, 2006.

[30] A. R. Teel, A. Subbaraman, and A. Sferlazza, "Stability analysis for stochastic hybrid systems: A survey," Automatica, vol. 50, no. 10, pp. 2435-2456, 2014.

[31] M. Soltani and A. Singh, "Moment-based analysis of stochastic hybrid systems with renewal transitions," Automatica, vol. 84, pp. 62-69, 2017.

[32] Z. Vahdat, Z. Xu, and A. Singh, "Modeling protein concentrations in cycling cells using stochastic hybrid systems," IFAC-PapersOnLine, vol. 54, no. 9, pp. 521-526, 2021.

[33] M. Soltani and A. Singh, "Moment analysis of linear time-varying dynamical systems with renewal transitions," SIAM Journal on Control and Optimization, vol. 57, no. 4, pp. 2660-2685, 2019.

[34] Z. Vahdat, Z. Xu, and A. Singh, "Modeling and characterization of neuronal synapses using stochastic hybrid systems," in 2019 IEEE 58th Conference on Decision and Control (CDC). IEEE, 2019, pp. 4729-4734.

[35] A. R. Teel and J. P. Hespanha, "Stochastic hybrid systems: A modeling and stability theory tutorial," in 2015 54th IEEE Conference on Decision and Control (CDC). IEEE, 2015, pp. 3116-3136.

[36] A. A. Julius and A. D'Innocenzo, "Combining analytical technique and randomized algorithm in safety verification of stochastic hybrid systems," in 2014 American Control Conference. IEEE, 2014, pp. $1438-1443$.

[37] H. Wang, P. Shi, C.-C. Lim, and Q. Xue, "Event-triggered control for networked markovian jump systems," International Journal of Robust and Nonlinear Control, vol. 25, no. 17, pp. 3422-3438, 2015.

[38] E.-Y. Kang, D. Mu, L. Huang, and Q. Lan, "Model-based analysis of timing and energy constraints in an autonomous vehicle system," in 2017 IEEE International Conference on Software Quality, Reliability and Security Companion (QRS-C). IEEE, 2017, pp. 525-532.

[39] C. A. Vargas-Garcia, M. Soltani, and A. Singh, "Conditions for cell size homeostasis: a stochastic hybrid system approach," IEEE Life Sciences Letters, vol. 2, no. 4, pp. 47-50, 2016.

[40] D. Antunes, J. P. Hespanha, and C. Silvestre, "Volterra integral approach to impulsive renewal systems: Application to networked control," Automatic Control, IEEE Transactions on, vol. 57, pp. 607 $-619,2012$.

[41] — "Stochastic hybrid systems with renewal transitions: Moment analysis with application to networked control systems with delays," SIAM Journal on Control and Optimization, vol. 51, pp. 1481,1499, 2013

[42] A. Singh and J. P. Hespanha, "Models for multi-specie chemical reactions using polynomial stochastic hybrid systems," in Proceedings of the 44th IEEE Conference on Decision and Control. IEEE, 2005, pp. 2969-2974.

[43] J. P. Hespanha and A. Singh, "Stochastic models for chemically reacting systems using polynomial stochastic hybrid systems," International Journal of Robust and Nonlinear Control, vol. 15, pp. 669-689, 2005.

[44] A. Singh and J. P. Hespanha, "Approximate moment dynamics for chemically reacting systems," IEEE Transactions on Automatic Control, vol. 56, pp. 414-418, 2011.

[45] M. Finkelstein, "Failure Rate and Mean Remaining Lifetime," in Failure Rate Modelling for Reliability and Risk, ser. Springer Series in Reliability Engineering. Springer, 2008, pp. 9-44.
[46] A. Singh and P. Bokes, "Consequences of mRNA transport on stochastic variability in protein levels," Biophysical Journal, vol. 103, pp. 1087-1096, 2012.

[47] M. Soltani, P. Bokes, Z. Fox, and A. Singh, "Nonspecific transcription factor binding can reduce noise in the expression of downstream proteins," Physical Biology, vol. 12, p. 055002, 2015.

[48] J. C. Maxwell, "V. illustrations of the dynamical theory of gases.part i. on the motions and collisions of perfectly elastic spheres," The London, Edinburgh, and Dublin Philosophical Magazine and Journal of Science, vol. 19, no. 124, pp. 19-32, 1860.

[49] H. D. Young, R. A. Freedman, and A. L. Ford, "University physics with modern physics vol. 2," 2008.

[50] K. R. Ghusinga, M. Soltani, A. Lamperski, S. Dhople, and A. Singh, "Approximate moment dynamics for polynomial and trigonometric stochastic systems," Proc. of the 56th IEEE Conf. on Decision and Control, Melbourne, 2017.

[51] Z. Konkoli, "Modeling reaction noise with a desired accuracy by using the X level approach reaction noise estimator (XARNES) method," Journal of Theoretical Biology, vol. 305, pp. 1-14, 2012.

[52] P. Smadbeck and Y. N. Kaznessis, "A closure scheme for chemical master equations," Proceedings of the National Academy of Sciences, vol. 110, pp. 14261-14 265, 2013.

[53] M. Soltani, C. Vargas, and A. Singh, "Conditional moment closure schemes for studying stochastic dynamics of genetic circuits," IEEE Transactions on Biomedical Systems and Circuits, vol. 9, pp. 518-526, 2015

[54] K. R. Ghusinga, C. A. Vargas-Garcia, A. Lamperski, and A. Singh, "Exact lower and upper bounds on stationary moments in stochastic biochemical systems," Physical biology, vol. 14, no. 4, p. 04LT01, 2017.

[55] K. R. Ghusinga, A. Lamperski, and A. Singh, "Moment analysis of stochastic hybrid systems using semidefinite programming," Automatica, vol. 112, p. 108634, 2020

[56] C. Kuehn, "Moment closure-a brief review," in Control of SelfOrganizing Nonlinear Systems, ser. Understanding Complex Systems, E. Schöll, S. H. L. Klapp, and P. Hövel, Eds. Springer International Publishing, 2016, pp. 253-271.

[57] P. Milner, C. S. Gillespie, and D. J. Wilkinson, "Moment closure approximations for stochastic kinetic models with rational rate laws," Mathematical Biosciences, vol. 231, pp. 99-104, 2011.

[58] A. Lamperski, K. R. Ghusinga, and A. Singh, "Analysis and control of stochastic systems using semidefinite programming over moments," IEEE Transactions on Automatic Control, vol. 64, no. 4, pp. 17261731,2018

[59] R. Brown, "Xxvii. a brief account of microscopical observations made in the months of june, july and august 1827 , on the particles contained in the pollen of plants; and on the general existence of active molecules in organic and inorganic bodies," The philosophical magazine, vol. 4 no. 21 , pp. $161-173,1828$.

[60] D. M. Wilkinson, "Brown knew particles were smaller than pollen," Nature, vol. 434, no. 7030, pp. 137-137, 2005.

[61] Z. Vahdat and A. Singh, "Time triggered stochastic hybrid system with nonlinear continuous dynamics," 2021 